\title{
Visual Interpretation of Harry Potter Novels
}

\author{
Anna Dilanian \\ Yerevan State University
}

\begin{abstract}
This article seeks to explain the nature of visual interpretation which is an inseparable part of visual linguistics. Within the frames of this article we intend to study and illustrate the techniques that are used for making any kind of visual interpretation. To be more practical, illustrations and movie images have been used. The article focuses on the study of the two main characters of Harry Potter novels. The characters have been thoroughly analyzed with the method of triangle analysis.
\end{abstract}

Key words: visual interpretation, image, illustration, semiotics, linguistics.

\section{Introduction}

Nowadays, in linguistics there is a new interdisciplinary study called Visual Linguistics. The latter is quite a new approach to language sciences, which has originated in the USA and later been successfully developed in the UK and Germany. Some attempts have also been made in this new field of linguistics by Russian scholars.

In the article we intend to study two main Harry Potter characters and their visual interpretation. The study is carried out with the help of textual reference and visual analysis. The characters have been analyzed through the method of triangle analysis which in fact is the relation of text, illustration and image. One of our main goals is to show that being an interdisciplinary study, visual linguistics has a lot to do with other disciplines, thus extra factors reflect on the choice of any kind of visual technique. 


\section{The Nature of Visual Linguistics}

Visualization as a means of illustration has a long tradition in linguistics. Starting from the antique times up to modern writers, literature as a separate form of art is the primary basis for many other branches of art: painting, music, theatre and cinema. Visual interpretation of literature is realized with the help of visual means: drawings, illustrations, photos and so on. There may be different visual interpretations of the same well-known literary work; such a case is Harry Potter.

What is worth mentioning is that visual interpretation as such is individual: the artist expresses his/her own understandings of a literary work, though the general idea is clearly kept. As Cristopher Orr remarks: "Within this critical context [i.e. of intertextuality], the issue is not whether the adapted film is faithful to its source, but rather how the choice of a specific source and how the approach to that source serve the film's ideology" (Orr 1984:86).

The concept of "movie interpretation" can be explained as a creative search for expressing the works of literature by means of cinematic decisions. Nowadays, books are considered to be a source of movie interpretations and the latter consequently became a separate form of art i.e. filmmaking. Nowadays, cinematographic interpretations are popular not as a separate form of art but as a tool for propagating literature as such. However, considering all the modern forms of interpretation of literary works, it can be noted that literature, as a separate form of art, is destined to become a fundamental source for many other forms of art.

Visual linguistics is closely interrelated with multimodality and semiotics. The former, in the most general sense, is the theory of communication and social semiotics. The most important study in this field is realized by Halliday who gives definitions for both multimodality and semiotics. Interestingly enough he thinks that the mixture of these two phenomena is the most effective contribution to the study of visual linguistics.

Multimodality describes the process of communication in terms of textual, auditory, linguistic, spatial and visual resources or other modes that are used for transferring the linguistic message. Another study which is the fundamental 
base of visual linguistics, as mentioned above, is semiotics. Semiotics is the theory of sign systems. "For decades it has been assumed that every sign system in nature, society and cognition belongs to the domain of semiotics. The literature on the subject is voluminous" (Nazarova 1996:79). No matter how different the signs are, all of them have a common nature: they are socialized, from era to era and from one society to another they change radically.

The above mentioned proves the fact that visual interpretation varies from culture to culture: things that are accepted for one culture may be quite harmful and inappropriate for another, so with the aim to be delicate and not to offend the representatives of the supposed culture visual interpretation must be accomplished very delicately. Hence, all of these give us the right to conclude that some extra factors reflect on the process of visualization. One of them, as mentioned above, is cultural differences, hence we can state that visual interpretation is a very subtle process. Another factor is the question of gender studies. Nowadays, gender studies are an integral part of both social sciences and linguistics in general, and, as already mentioned, visual linguistics is somewhere in between these two disciplines, so the problem of gender, at the same time is the vital problem of visual linguistics, too.

Thus, any kind of visual interpretation follows one aim: to be aesthetic and allure large audiences. Aiming to be more delicate, the illustrator and/or the director are allowed to add or omit things in his/her interpretation.

\section{Characters' Visual Interpretation}

The first character that is described in this article is the main protagonist of the book - Harry Potter. Harry is described in the following way: "Harry had always been small and skinny for his age. ... Harry had a thin face, knobby knees, black hair, and bright green eyes. He wore round glasses held together with a lot of Scotch tape.... The only thing Harry liked about his own appearance was a very thin scar on his forehead that was shaped like a bolt of lightning" (Rowling 1994:47). In the movies Harry appears with blue eyes and is not skinny at all. While asking the directors why there were such short-cuts, the reply was that among all the candidates the actor who portrayed Harry was 
the most similar one. As a matter of fact the question of green and blue eyes was a serious problem for the most faithful fans. In an illustrated version of Harry Potter which was approved by the author, Harry had green eyes. Below are the pictures of Harry Potter taken from the illustrated version of the book and the one from the movie.

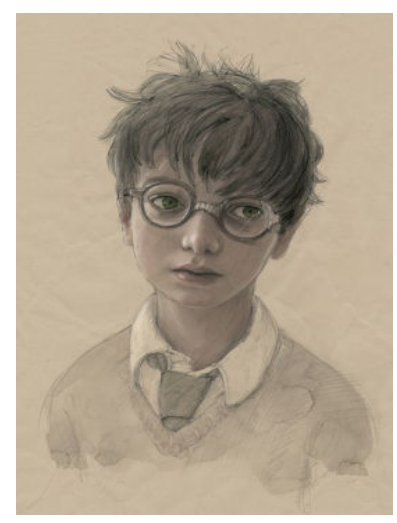

(Kay 2018)

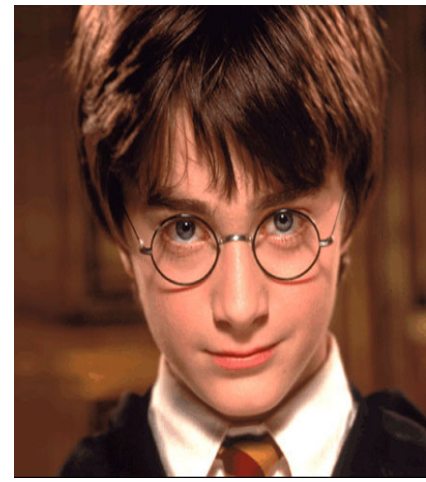

(WB 2001)

As can be seen, the clothes and attributes (exactly the way Rowling described) are all preserved in both versions but the appearance is somehow altered.

The second character is Hermione Granger. In the books Hermione Granger is described as having a bossy sort of voice, lots of bushy brown hair, and rather large front teeth" (Rowling 1997:105). The point that was a huge argument between the fans was the fact of Hermione not having large teeth in the movie. The director of the first Harry Potter movies Chris Columbus revealed that they initially tried to use fake teeth but it was uncomfortable and affected the speech. So from the point of view of visual linguistics this is an unfaithful interpretation. Bellow you can see Hermione's illustrated version and the movie Hermione. 


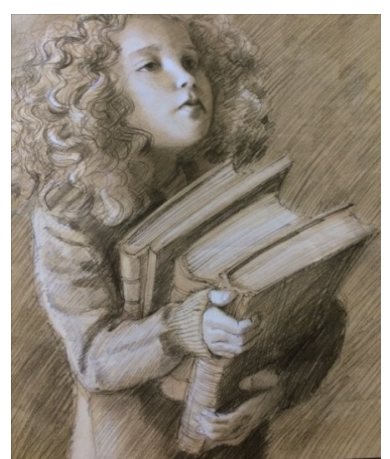

(Kay 2018)

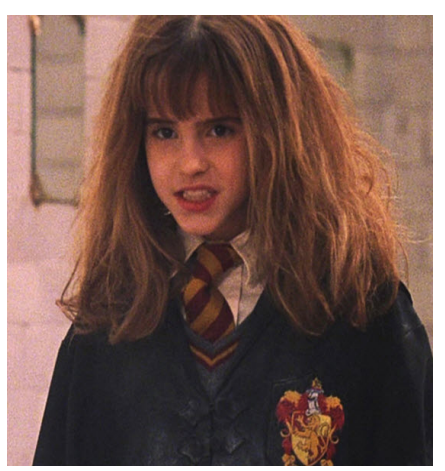

(WB 2001)

As mentioned above, some extra factors can affect the choice of this or that way of visual interpretation. In all the book series, there is no mention of the color of Hermione's clothes but in the third book she appears in pink clothes; this is a stereotypical phenomenon: in all cultures pink is considered to be girlish. This comes to prove the fact that visual linguistics as such is an interdisciplinary study and closely associated with newly developed gender studies. Another example of it is the following. At the Yule Ball, Hermione was wearing a blue dress: "She had done something with her hair; it was no longer bushy but sleek and shiny, and twisted up into an elegant knot at the back of her head. She was wearing robes made of a floaty, periwinkle-blue material, and she was holding herself differently, somehow..." (Rowling 2000:117). Whereas in the movie she appears in a pink dress.

The character's visual interpretation is more likely to be not faithful to the source material but more aesthetic, whereas the book cover and movie poster strongly reflect the text. The latters are so close to the source material that may even contain spoilers and allow the reader or viewer make some guesses even without reading the book. Another reason why the character's visual interpretation is not faithful to the text is that the directors sometimes consciously cut the flaws in the character's appearance, as many directors mention: "It is because people love watching beautiful actors on the screen" (Vogue 2007:34). 


\section{Conclusion}

Visual interpretation which has been studied in terms of two main characters was aimed at showing gender roles. The scholars in the field of visual linguistics differentiate between two types of visual interpretation: faithful interpretation and adoptive interpretation. The book covers and movie posters are mostly interpreted faithfully, whereas characters are adoptive, as both actors and illustrations cannot be completely like the source prototype.

Visual linguistics is closely connected with disciplines like social sciences, humanities and newly developed gender studies. Thus, while making any kind of visual interpretation every single detail is taken into consideration and, what is more important, visual interpretation is individual. Such cases are Hermione's clothes and Harry's appearance.

Any kind of visual interpretation is done on purpose and thus varies from culture to culture with the aim of not being harmful. The illustrators' task is to be delicate as, without even understanding, one can offend others' feelings. Hence visual linguistics mostly deals with philosophical and psychological aspects.

\section{References:}

1. Rozhdestvenskaya Ye. (2001) Kachestvenniye metody v gendernoy metodologii. // Genderniy kaleydoskop. M.: Izd-vo ISEPN.

2. Rozhdestvenskaya Ye. (2001) Feministskiy podkhod k interpretatsii kachestvennykh dannykh: metody analiza teksta, interaktsii $i$ izobrazheniya. // Vvedenie v gendernye issledovaniya. S.-Peterburg: Aleteyya.

3. Nazarova T. (1996) Linguistics and Literary Semiotics. M.: MSU.

4. Orr C. (1984) The Discourse on Adaptation. New York: Wide Angle.

\section{Sources of Data:}

1. Rowling, J. (1997) Harry Potter and the Philosopher's Stone. London: Bloomsbury.

2. Rowling, J. (1998) Harry Potter and the 'Chamber of Secrets' London: Bloomsbury. 
3. Rowling, J. (1999) Harry Potter and the Prisoner of Azkaban. London: Bloomsbury.

4. Rowling, J. (2000) Harry Potter and the Goblet of fire. London: Bloomsbury.

5. (2001) Harry Potter and the Philosopher's Stone, film USA.

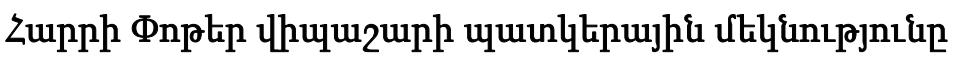

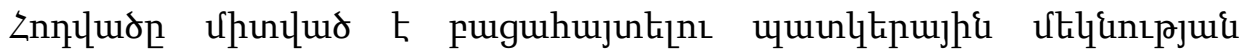

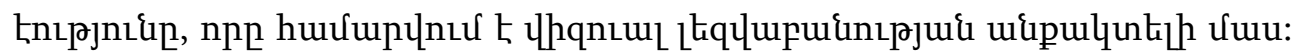

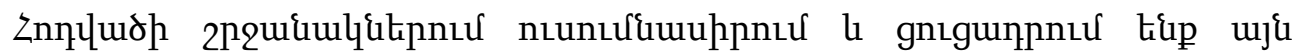

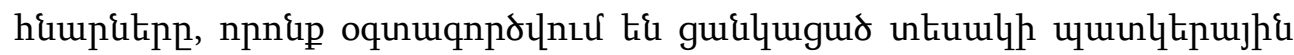

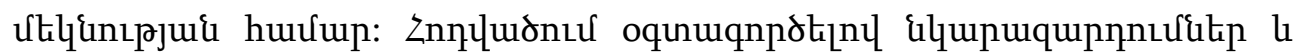

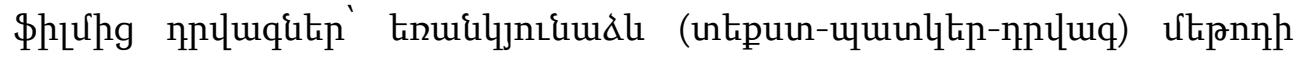

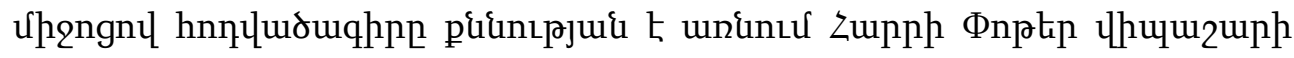

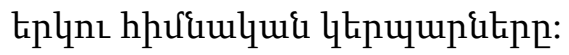

